\title{
A RARE CAUSE OF SEIZURES WITH MENTAL RETARDATION AND HEMIPARESIS-DYKE-DAVIDOFF-MASSON SYNDROME
}

\author{
Vipin Kumar Bakshi ${ }^{1}$, Hemant Kumar Mishra², Sunny Goyal ${ }^{3}$
}

\section{HOW TO CITE THIS ARTICLE:}

Vipin Kumar Bakshi, Hemant Kumar Mishra, Sunny Goyal. "A Rare Cause of Seizures with Mental Retardation and Hemiparesis-Dyke-Davidoff-Masson Syndrome". Journal of Evolution of Medical and Dental Sciences 2014; Vol. 3, Issue 48, September 29; Page: 11631-11638, DOI: 10.14260/jemds/2014/3527

ABSTRACT: Cerebral hemi-atrophy with seizures, hemiplegia and mental retardation is uncommon clinical presentation in the early childhood and adolescence. The causes are various and usually grouped into congenital and acquired types. The Dyke-Davidoff-Masson Syndrome is one of the causes of these clinical manifestations, which is usually developed secondary to brain insult. Here we report two cases with similar symptoms- one case was an adult male of 35 years with long history of seizures and the second case was a young male of 22 years with cognitive impairment, difficulty in speech and walking.

KEYWORDS: DDMS-Dyke Davidoff Masson Syndrome, Cerebral hemi-atrophy, MRI-Magnetic Resonance Imaging, CT-Computerized Tomography.

INTRODUCTION: Dyke-Davidoff-Masson Syndrome is one of the causes of seizures with mental retardation. It was first described by C.G. Dyke, L.M. Davidoff and C. B. Masson in 1933 on plain skull radiograph and pneumatoencephalogram in a series of nine patients. The plain skull radiograph shows calvarial thickening with ipsilateral dilatation of paranasal sinuses. ${ }^{3}$ In 1939, Alpers and Deer defined two types of Cerebral hemiatrophy- Congenital and Acquired. ${ }^{1}$ The Congenital types show hypo plastic cerebral cortex with thickened calvaria. The Acquired type is usually from the cerebrovascular lesion and inflammatory processes.

Hageman et al in his study described cerebral atrophy, is actually a lack of cerebral development rather than atrophy. ${ }^{5}$ The brain reaches half of its adult size during first year of life and three-fourth by the end of third year. ${ }^{11}$ As the brain enlarges, it compresses the inner table of calvaria. When the brain is not developing, the bony structures grow inward causing dilatation of paranasal sinuses and flattening of calvarium. Overpneumatization of paranasal sinuses and mastoid air cells lead to elevation of the petrous ridge. (Jacoby et al., 1997)

Etiology of Congenital type is brain insult secondary to intrauterine vascular occlusion or Malformation in-utero and in neonatal period. This cause includes occlusion of Middle Cerebral Artery, Unilateral Cerebral Arterial circulation, Co-arctation of mid-aortic arch, mesencephalon hyperplasia etc. ${ }^{10,8,4}$

Acquired variety includes Birth trauma, Perinatal intracranial haemorrhages, Rasmussen encephalitis, Post-ictal cerebral hemiatrophy, Infection like Herpes Encephalitis, Vascular abnormalities like Dyke-Davidoff-Masson Syndrome, Sturge-Weber Syndrome, Ischaemia, Neoplasia like Basal cell germinoma, Radiation, Phakomatosis (Neurofibromatosis) etc. ${ }^{4}$ Plain X-ray skull, Computerized Tomography (CT) and Magnetic Resonance Imaging (MRI) are helpful in diagnosis of DDMS. The MRI is preferred modality for the assessment of etiology and lesion extent of cerebral parenchyma. $^{2}$ 
CASE REPORTS: CASE 1: A 22 years old male patient presented to the Department of Medicine with mental retardation and right hemiplegia since 18 years. The patient also complained of difficulty in speech, cognitive impairment and difficulty in walking. There is no history of seizure disorder.

PHYSICAL AND CLINICAL EXAMINATION: Patient was a young male, moderately built and nourished\& disoriented to time, place and person with irrelevant talking. His general physical examination and vitals were normal. Central Nervous System examination was normal. Examination of other systems was unremarkable. His routine blood and urine investigations were normal Radiological Examination.

Skiagram of Skull (AP \& Lateral views) revealed osseous hypertrophy of calvarium on left side (Ipsilateral). No evidence of increased intra-cranial tension or abnormal calcification noted. Sutures were normal. Skiagram of Para Nasal air Sinuses revealed hypertrophy and hyper-pneumatization of left (Ipsilateral) paranasal air sinuses, predominantly, frontal, maxillary and ethmoid sinuses.

NCCT of Brain revealed hemi-atrophy of left cerebral hemisphere, leading to dilatation of sulci and cisterns as well as ex-vacuo dilatation of left lateral ventricle, atrophy of left cortico-spinal tract/cerebral peduncle in left half of mid-brain suggestive of wallerian degeneration. Due to volume loss, there is compensatory thickening of left frontal, parietal and squamous part of left temporal bone with enlargement of left frontal sinus and ethmoid sinus.

Para nasal air sinuses showed hypertrophy and hyper-pneumatization of left (Ipsilateral) paranasal air sinuses, predominantly, frontal, maxillary and ethmoid sinuses. MRI of Brain revealed marked atrophy of left cerebral hemisphere with prominent sylvian fissure and sulci. There are gliotic and encephalomalacic changes in the left cerebral hemisphere with compensatory hypertrophy of right cerebral hemisphere with displacement of falx to left side.

There is compensatory thickening of skull vault with enlargement of left frontal and ethmoidal sinuses, enlarged left mastoid air cells with elevation of petrous part of left temporal bone. No evidence of calcification noted. The left anterior, middle and posterior cerebral arteries appear small in caliber than right.

Fig. 1: T1 Axial Section Showing Left Cerebral Hemiatrophy and Thickening of Ipsilateral Calvarium with Hyperpneumatization of Left Frontal Sinus.

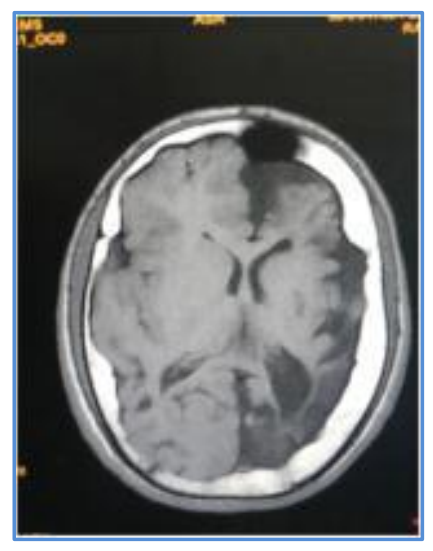

Figure 1 


\section{CASE REPORT}

Fig. 2: T2- Flair Axial Section Showing Hyperpneumatization of Left (Ipsilateral) Frontal Sinus.

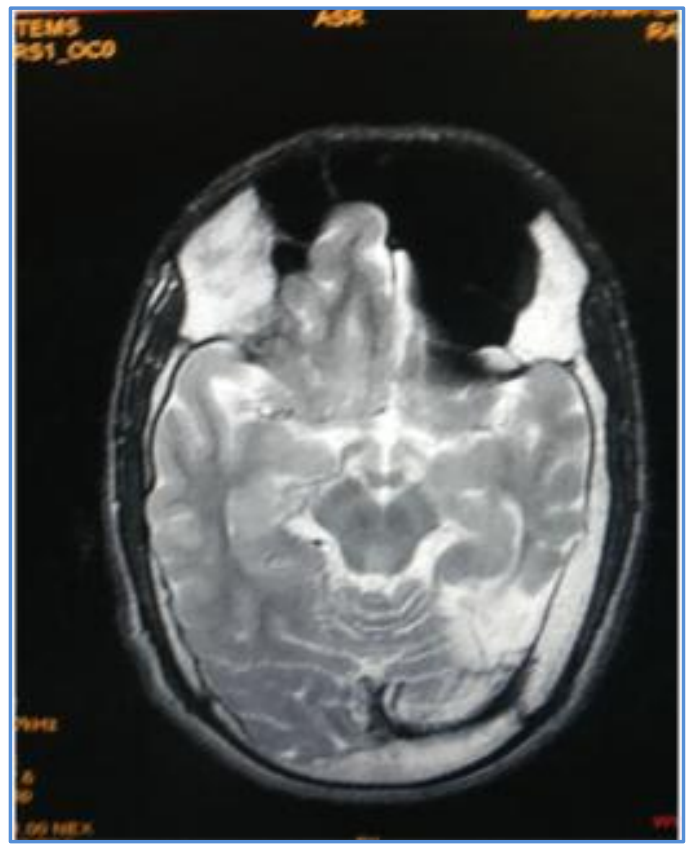

Figure 2

Fig. 3: T2-Flair Axial Section Showing Left Cerebral Hemiatrophy and Thickening of Ipsilateral Calvarium with Hyperpneumatization of Left Frontal Sinus.

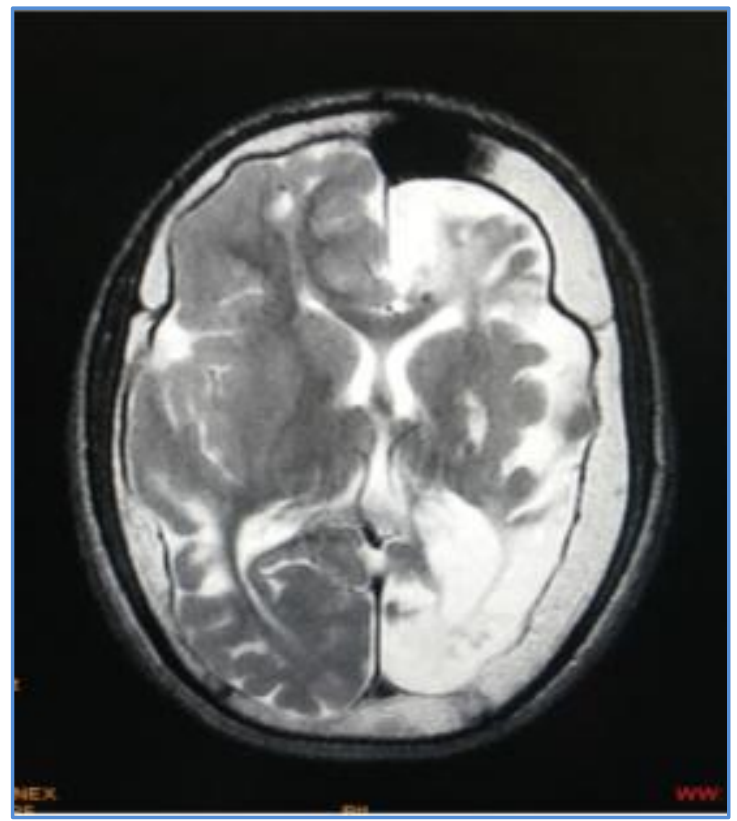

Figure 3 
The Colour Doppler Study of Left CCA and Left ICA are reduced in caliber with normal flow and indices. There is evidence of diastolic dip with reversal of flow in left CCA. Both Vertebral arteries, Right CCA and ICA are normal in caliber and flow.
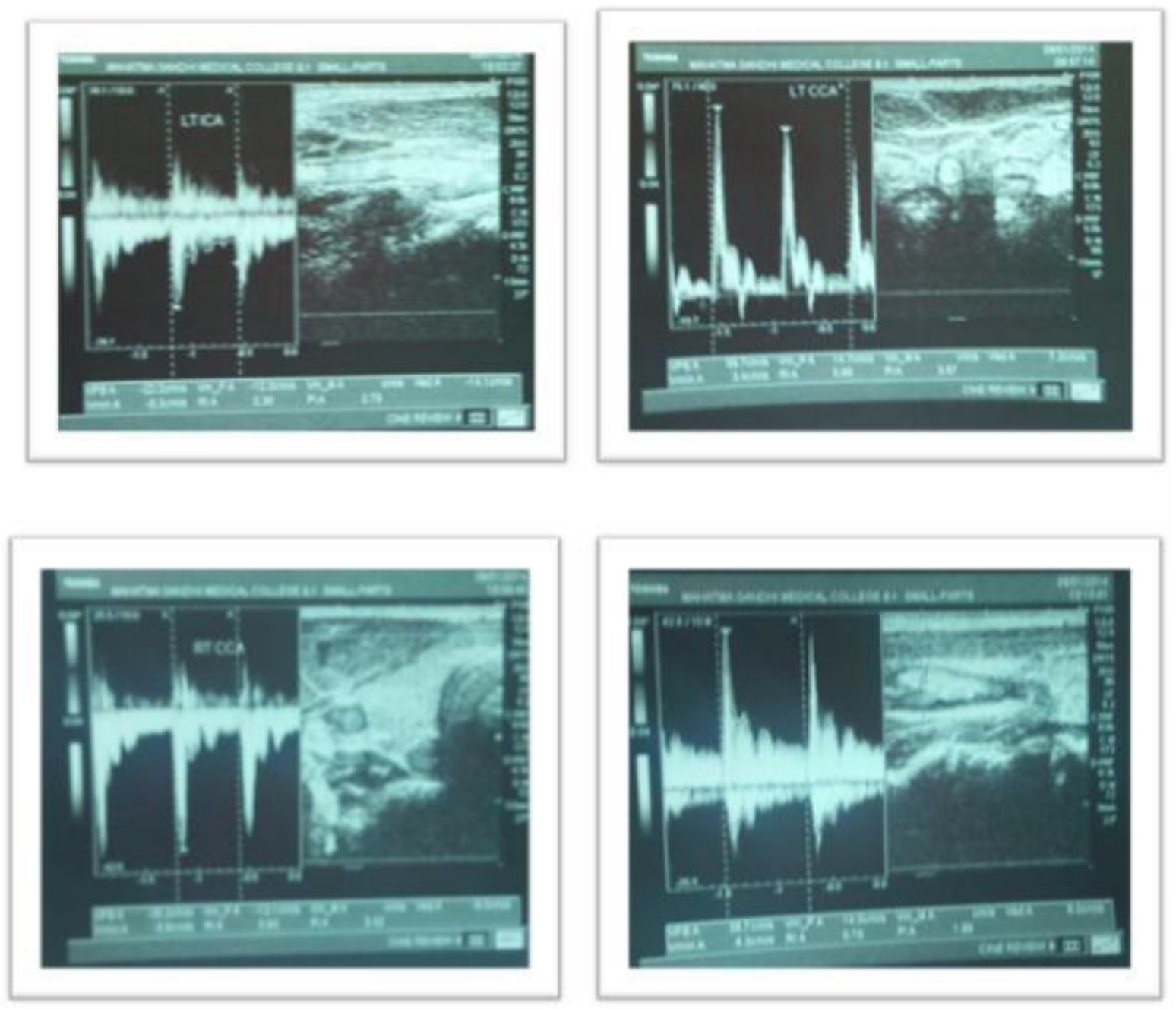

\section{COLOUR DOPPLER EVALUATION OF CAROTID VESSELS}

CASE 2: A 35 years old male patient presented to the Department of Casualty with Complaints of involuntary movements of both upper and lower limbs with frothing from mouth since 1 day, associated deviation of angle of mouth to the right side. The patient gives history of several such episodes in past, with each episode lasting for approximately 5 minutes with loss of consciousness. These episodes started at the age of 7 years.

Patient was a known case of seizure disorder and had taken medication intermittently. There was no control of seizures even on medication. Patient was also given some native medicines but with no improvement in symptoms. Despite all the above efforts, he was getting seizures regularly. Patient's birth history was uneventful. However, patient's developmental history was significant with delayed milestones and mental retardation. Patient had not done any schooling, so far. There is no $\mathrm{h} / \mathrm{o}$ any serious childhood infections, neuro-infection or any trauma. 
PHYSICAL AND CLINICAL EXAMINATION: Patient was a young male, moderately built and nourished\& disoriented to time, place and person with irrelevant talking. His general physical examination and vitals were normal. Central Nervous System examination revealed only hypotonia with exaggerated deep tendon reflexes and bilateral extensor plantar response along with fanning of toes. Examination of other systems was unremarkable.

RADIOLOGICAL EXAMINATION: Skiagram of Skull (AP \& Lateral views) revealed osseous hypertrophy of calvarium on right side (Ipsilateral). No evidence of increased intra-cranial tension or abnormal calcification noted. Sutures were normal.

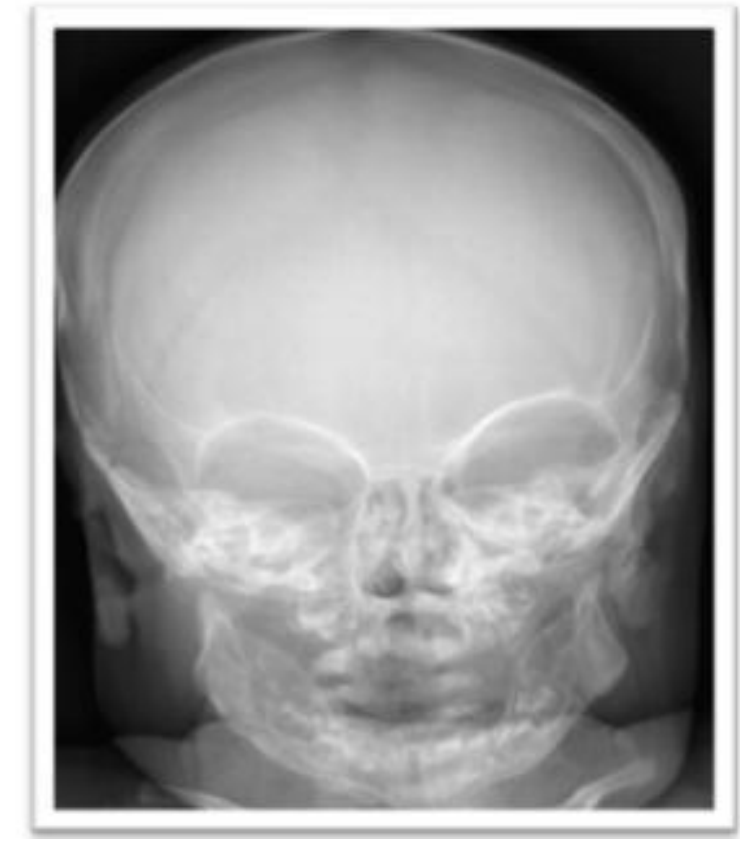

SKULL RADIOGRAPH - AP VIEW

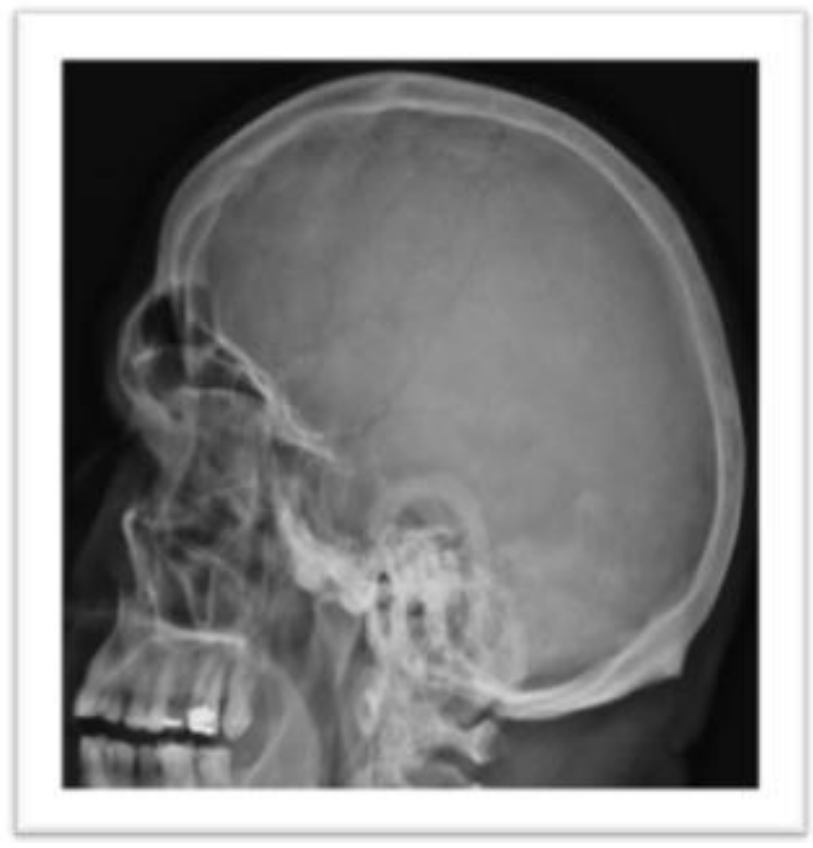

SKULL RADIOGRAPH - AP VIEW

AP AND LATERAL SKULL RADIOGRAPHS OF PATIENT WITH DDMS

Skiagram of Para Nasal air Sinuses revealed hypertrophy and hyper-pneumatization of right (Ipsilateral) paranasal air sinuses, predominantly, frontal, maxillary and ethmoid sinuses. Computerized Tomography of the Brain revealed hemi-atrophy of right cerebral hemisphere, leading to dilatation of sulci and cisterns as well as ex-vacuo dilatation of right lateral ventricle, atrophy of right cortico-spinal tract/cerebral peduncle in right half of mid-brain suggestive of wallerian degeneration.

Due to volume loss, there is compensatory thickening of right frontal, parietal and squamous part of right temporal bone with enlargement of right frontal sinus and ethmoid sinus. Para nasal air sinuses showed hypertrophy and hyper-pneumatization of left (Ipsilateral) paranasal air sinuses, predominantly, frontal, maxillary and ethmoid sinuses. 


\section{CASE REPORT}

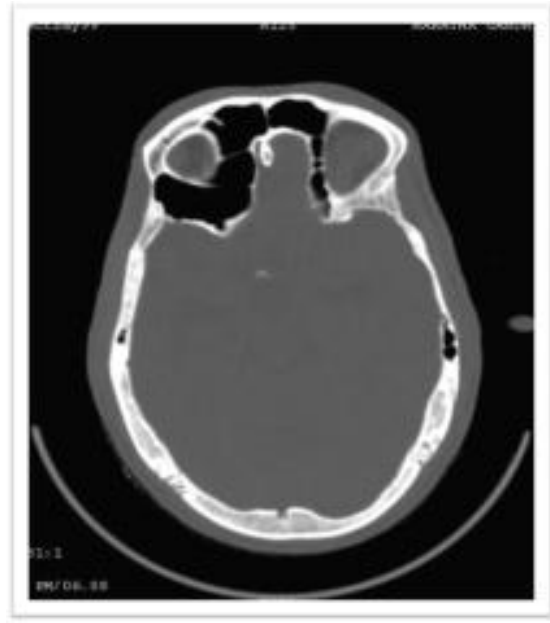

NCCT Brain Showing Osseous Hypertrophy of Calvarium on Right (Ipsilateral) Side

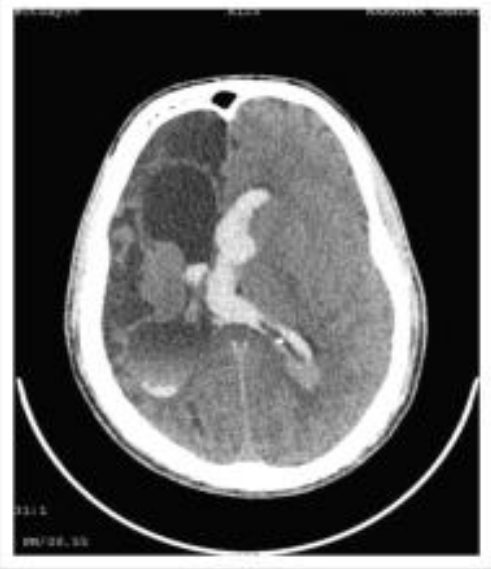

CT Brain (Axial Section) Showing Hemi-Atrophy of Right Cerebral Hemisphere, Leading to Ex-Vacuo Dilatation of Right Lateral Ventricle

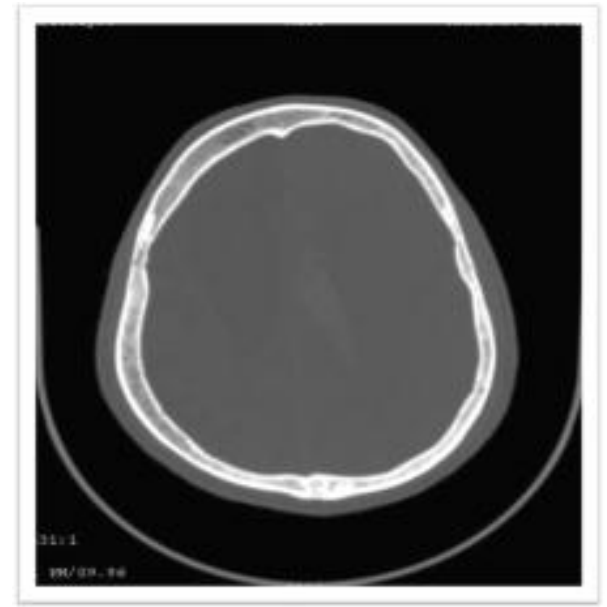

Axial Ct Section (Bone Window) Showing Osseous Hypertrophy of Calvarium on Right (Ipsilateral) Side

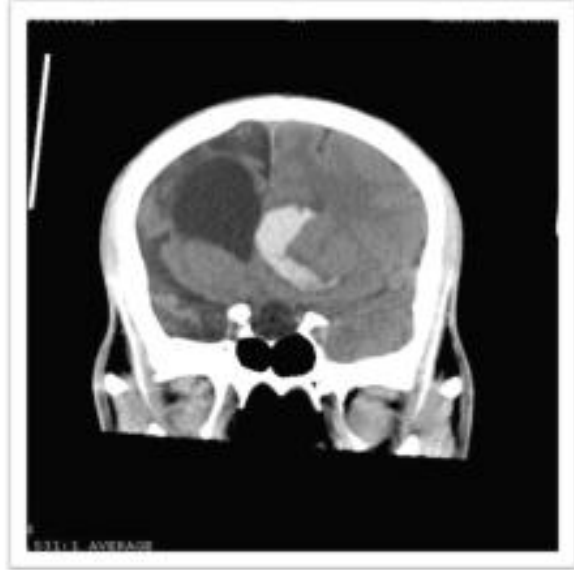

CT Brain (Coronal Section) Showing Right Cerebral Hemiatrophy

DISCUSSION: Cerebral hemi-atrophy is an entity with diverse etiologies and pronounced asymmetry of cerebral hemispheres. ${ }^{12}$ It may have progressive spectrum from the single event or emanating from a single event. ${ }^{9}$ Atalar et al detected left cerebral predominance in 14 out of 19 cases studied. ${ }^{2}$ DDMS is clinically characterized by hemiparesis/hemiplegia, seizures, facial asymmetry and mental retardation. ${ }^{10,11}$

The radiological and imaging features include ipsilateral osseous hypertrophy, hyperpneumatization of paranasal sinuses and mastoid air cells and cerebral hemiatrophy with mild compensatory hypertrophy of contralateral sulcus. 
The exact mechanism of cerebral atrophy is still unclear, but it is hypothesized that ischemic insult to brain from various causes reduce the production of the neurotropic factors which lead to atrophy. ${ }^{6}$

The Differential Diagnosis of cerebral hemiatrophy with hemiplegia and seizures includes Herpes Simplex Encephalitis, Sturge-Weber syndrome, Rasmussen encephalitis, Crossed Cerebellar Diaschisis, Perinatal asphyxia, Parry-Romberg syndrome, Linear Sebacious Nevus Syndrome, Linear Scleroderma, Status Epilepticus, Post-Ictal cerebral hemiatrophy, Neurofibromatosis, Head trauma, Brain tumours etc.

Herpes Simplex Encephalitis (HSE) is a non-epidermic, necrotizing meningo-encephalitis with haemorrhagic necrotic tendency, considerable mass effect and rapid dissemination in brain. SturgeWeber Syndrome (SWS) or Encephalo-trigeminal Angiomatosis is a phakomatosis. It is characterized by facial port wine stains and pial angiomas.

Rasmussen Encephalitis (RE) or Chronic Focal Encephalitis is a chronic inflammatory disease of unknown origin, usually affecting one cerebral hemisphere. Crossed Cerebellar Diaschisis (CCD) refers to a depression of blood flow and metabolism affecting the cerebellar hemisphere occurring as a result of a contralateral focal, supra-tentorial lesion.

Parry-Romberg syndrome (PRS) or Progressive Facial Hemiatrophy is classically characterized by a slow progressive degeneration (atrophy) of the soft tissues of half of the face (hemifacial atrophy).

This involves the skin, underlying soft tissues, cartilaginous structures and bone. Linear Sebacious Nevus Syndrome is a neurocutaneous condition similar to tuberous sclerosis with mental retardation, convulsions and characteristic linear nevus of the face.

CONCLUSION: DDMS can be diagnosed with detailed clinical history, skull radiograph and neuroimaging modalities like CT and MRI. The treatment is usually symptomatic and includes management of seizures, hemiparesis and others. Prognosis is better if hemiparesis occurs after the age of two years.

\section{REFERENCES:}

1. Alpers B J and Dear R B, Hemi atrophy of the brain, J Nerv Ment Dis 1939; 89: 653-651.

2. Atalar MH, Icagasioglu D, Tas F. Cerebral hemi atrophy (Dyke-Davidoff-Masson Syndrome) in childhood: clinic-radiological analysis of 19 cases. Pediatr Int.2007 Feb; 49 (1): 70-7.

3. Dyke C.G., Davidoff L.M., Masson C.B. Cerebral Hemi atrophy and homo lateral hypertrophy of the skull and sinuses. Surg Gynecol Obstet. 1933; 57: 588-600.

4. Goyal J, Shah V, Rao S, Jindal N. Dyke-Davidoff-Masson Syndrome in Children. The Internet Journal of Pediatrics and Neonatology. 2009; 10 (2): 101-107.

5. Hageman G, Gooskens R H J M, Willemse J, A cerebral cause of arthrogryposis: Unilateral cerebral hypoplasia. Clin Neurol Neurosurg. 1985; 87: 119-122.

6. Lee JH, Lee ZI, Kim HK, Kwon SH. A case of Dyke-Davidoff-Masson Syndrome in Korea. Korean Journal of Paediatrics. 2006; 49 (2): 208-211.

7. Narain NP, Kumar R, Narain B. Dyke-Davidoff-Masson Syndrome. Indian Pediatrics. 2008; 45: 927-928.5.

8. Pendse NA, Bapna P, Menghani V, Diwan A. Dyke-Davidoff-Masson Syndrome. (DDMS). Ind J Paed.2004; 71: 943. 


\section{CASE REPORT}

9. Porettis, A., Wolfab, N. I., \& Boltsgausera, E. (2008). Differential diagnosis of cerebellar atrophy in childhood. European Journal of Paediatric neurology, 12, 155-167.

10. Sharma S, Goyal D, Negi A, Sood RG, Jhobta A, Surya M. Dyke-Davidoff-Masson Syndrome. Ind J Radiol Imag. 2006; 16 (2): 165-166.

11. Singh, J. P., Shrimali, R, Garg, L. \& Setia, V. (2002). Radiological quiz - brain. Indian J Radiol Imaging, 12, 439-40.

12. Vosskamper, M., \& Schachenmayr, W. (1990). Cerebral hemiatrophy: a clinic pathological report of two cases with a contribution to pathogenesis and differential diagnosis. Clin Neuropathol, 9 (5), 244-50.

\section{AUTHORS:}

1. Vipin Kumar Bakshi

2. Hemant Kumar Mishra

3. Sunny Goyal

\section{PARTICULARS OF CONTRIBUTORS:}

1. Post Graduate Resident, Department of Radio diagnosis, Mahatma Gandhi Medical College \& Hospital, Jaipur, Rajasthan.

2. Professor \& HOD, Department of Radio diagnosis, Mahatma Gandhi Medical College \& Hospital, Jaipur, Rajasthan.

3. Post Graduate Resident, Department of Radio diagnosis, Mahatma Gandhi Medical College \& Hospital, Jaipur, Rajasthan.

\section{NAME ADDRESS EMAIL ID OF THE CORRESPONDING AUTHOR:}

Dr. Vipin Kumar Bakshi, C/o Dr. V. K. Bakshi, Bakshi Niwas, 89-B Block, Sri Ganganagar, Rajasthan-335001.

Email: drvipinbakshi@yahoo.com

Date of Submission: 09/09/2014.

Date of Peer Review: 10/09/2014.

Date of Acceptance: 20/09/2014.

Date of Publishing: 29/09/2014. 